\title{
A global result for discrete $\phi$-Laplacian eigenvalue problems
}

Dingyong Bai*

\author{
Correspondence: \\ baidy@gzhu.edu.cn \\ School of Mathematics and \\ Information Science, Key Laboratory \\ of Mathematics and \\ Interdisciplinary Sciences of \\ Guangdong Higher Education \\ Institutes, Guangzhou University, \\ Guangzhou, 510006, P.R. China
}

\begin{abstract}
Concerned is the existence, nonexistence and multiplicity of positive solutions for discrete $\phi$-Laplacian eigenvalue problems. By using lower and upper solutions method and fixed point index theory, a global result with respect to parameter is established.
\end{abstract}

Keywords: discrete $\phi$-Laplacian eigenvalue problem; positive solution; the lower and upper solutions method; fixed point index theory

\section{Introduction}

For $a, b \in \mathbf{Z}$ with $a<b$, let $[a, b]_{\mathbf{Z}}=\{a, a+1, a+2, \ldots, b-1, b\}$. In this paper, we consider the following discrete $\phi$-Laplacian eigenvalue problem

$$
\left\{\begin{array}{l}
\Delta(\phi(\Delta u(k-1)))+\lambda p(k) g(u(k))=0, \quad k \in[1, T]_{\mathrm{Z}} \\
u(0)=u(T+1)=0
\end{array}\right.
$$

where $T>1$ is a given positive integer, $\Delta u(k)=u(k+1)-u(k), \lambda$ is a positive parameter, $p:[1, T]_{\mathbf{Z}} \rightarrow(0, \infty)$ and $g: \mathbf{R}^{+} \rightarrow(0, \infty)$ is continuous. We assume that

(A1) $\phi: \mathbf{R} \rightarrow \mathbf{R}$ is an odd and strictly increasing homeomorphism;

(A2) $\lim _{u \rightarrow \infty} \frac{g(u)}{\phi(u)}=\infty$.

The function $\phi(u)$ covers two important cases: $\phi(u)=u$ and $\phi(u)=|u|^{p-2} u(p>1)$. If $\phi(u)=u$, then problem (1) is the classical second order difference Dirichlet boundary value problem. For the case that $\phi(u)=|u|^{p-2} u$, problem (1) is the well-known discrete $p$-Laplacian problem. The two cases have been widely studied. To name a few, see [1-8] and the references therein.

Problem (1) can be viewed as the discrete analogue of the following differential $\phi$ Laplacian problem

$$
\left\{\begin{array}{l}
\left(\phi\left(u^{\prime}\right)\right)^{\prime}+\lambda p(t) g(u(t))=0, \quad 0<t<1, \\
u(0)=u(1)=0,
\end{array}\right.
$$

which rises from the study of radial solutions for $p$-Laplacian equations $\left(\phi(u)=|u|^{p-2} u\right)$ on an annular domain (see [9], and references therein). Recently, the differential $\phi$-Laplacian problems have been widely studied in many different papers. We refer the readers to [1017] and the references therein.

\section{Springer}

(c) 2013 Bai; licensee Springer. This is an Open Access article distributed under the terms of the Creative Commons Attribution License (http://creativecommons.org/licenses/by/2.0), which permits unrestricted use, distribution, and reproduction in any medium, provided the original work is properly cited. 
For discrete $\phi$-Laplacian problems, there are less study results than differential $\phi$ Laplacian problems. See Cabada [18], Cabada and Espinar [19] and Bondar [20]. In [18, 19], some existence results were established by the upper and lower solutions method. In [20], the existence and uniqueness of solutions were discussed for mixed and Dirichlet boundary value problems by the fixed point theory of contraction mapping. To the best of our knowledge, there are no results on the existence and multiplicity of positive solutions for difference $\phi$-Laplacian problems. Therefore, the purpose of this paper is to establish a global result of positive solutions of (1). We state our main result as follows.

Theorem 1.1 Let (A1) and (A2) hold. Then there exists $\lambda^{*}>0$ such that problem (1) has at least two positive solutions for $\lambda \in\left(0, \lambda^{*}\right)$, at least one positive solution for $\lambda=\lambda^{*}$ and no solution for $\lambda>\lambda^{*}$.

The result is motivated mainly by the ideas in [11, 21], in which some global results of positive solutions were established for boundary value problems of $p$-Laplacian differential systems and $\phi$-Laplacian differential systems, respectively.

Generally, in order to make priori estimations on possible positive solutions of $\phi$ Laplacian problems, the function $\phi$ satisfies not only condition (A1), but also other additional conditions. For example, Wang [10] used the following condition.

$\left(\mathrm{A} 1^{*}\right)$ There exist two increasing homeomorphisms $\psi_{1}, \psi_{2}:(0, \infty) \rightarrow(0, \infty)$ such that for all $x$ and $y>0$,

$$
\psi_{1}(x) \phi(y) \leq \phi(x y) \leq \psi_{2}(x) \phi(y)
$$

In [21], a more general condition was given.

$\left(\mathrm{A1}^{* *}\right)$ For $\sigma>0$, there exists a constant $C_{\sigma}>0$ such that for all $s \in \mathbf{R}, \frac{\phi(\sigma s)}{\phi(s)}<C_{\sigma}$.

In our discussion for the single discrete problem (1), we only assume that $\phi$ satisfies condition (A1). In addition, in the discussion of nonlinear differential systems in [11, 21], monotonicity conditions were imposed on nonlinear terms. In this paper $g$ does not have to satisfy monotonicity conditions.

The remaining part of this paper is organized as follows. In Section 2, we show some lemmas for the later use. In Section 3, we show the proof of Theorem 1.1. Our proofs are mainly based on the upper and lower solutions technique arguments and the fixed-point index theory for cones.

\section{Some lemmas}

First, we introduce an existence result of solutions based on lower and upper solutions method for discrete $\phi$-Laplacian boundary value problems, which has been proved by Cabada [18].

Consider the following boundary value problem

$$
\left\{\begin{array}{l}
\Delta(\phi(\Delta u(k-1)))+f(k, u(k))=0, \quad k \in[1, T]_{\mathrm{Z}} \\
u(0)=u(T+1)=0
\end{array}\right.
$$

where 
(B1) $\phi: \mathbf{R} \rightarrow \mathbf{R}$ is an odd and strictly increasing function;

(B2) $f:[1, T]_{\mathbf{Z}} \times \mathbf{R} \rightarrow \mathbf{R}$ is continuous.

Let $E=\left\{u:[0, T+1]_{\mathbf{Z}} \rightarrow \mathbf{R}^{T+2}\right\}$ with the norm $\|u\|=\max _{t \in[0, T+1]_{\mathbf{Z}}}|u(t)|$. Given $u, v \in E$, we say that $u \leq v$ if $u(k) \leq v(k)$ holds for all $k \in[0, T+1]_{\mathbf{Z}}$.

Definition 2.1 $\alpha \in E$ is called a lower solution of problem (3) if

$$
\left\{\begin{array}{l}
\Delta(\phi(\Delta \alpha(k-1)))+f(k, \alpha(k)) \geq 0, \quad k \in[1, T]_{\mathrm{Z}} \\
\alpha(0) \leq 0, \quad \alpha(T+1) \leq 0
\end{array}\right.
$$

In a same way, we define the upper solution of (3) by reversing the above inequalities.

Lemma 2.1 [18] Let (B1) and (B2) hold. Assume that there exist $\alpha$ and $\beta$, respectively lower and upper solutions of (3) such that $\alpha \leq \beta$. Then problem (3) has at least one solution $u$ with $\alpha \leq u \leq \beta$.

A function $u$ of integer variable is said to be concave if $\Delta^{2} u(k-1) \leq 0$. This is the same as saying that the first difference $\Delta u(k-1)$ is non-increasing. If $\Delta^{2} u(k-1)<0$, then $u$ is said to be strictly concave.

Lemma 2.2 Let $u(k)$ be concave on $[0,1+T]_{\mathrm{Z}}$, and $u(0) \geq 0, u(T+1) \geq 0$. Then:

(i) $u(k) \geq 0$ for all $k \in[1, T]_{\mathrm{Z}}$, and $\Delta u(k-1) \geq 0$ for $k \in\left[1, k^{*}\right]_{\mathrm{Z}}, \Delta u(k) \leq 0$ for $k \in\left[k^{*}, T\right]_{\mathrm{Z}}$, where $k^{*} \in[0,1+T]_{\mathrm{Z}}$ satisfies $u\left(k^{*}\right)=\max _{k \in[0,1+T]_{\mathrm{Z}}} u(k)$.

(ii) For each $k_{0} \in\left[1,\left\lfloor\frac{T+1}{2}\right\rfloor-1\right]_{\mathbf{Z}}\left(\left\lfloor\frac{T+1}{2}\right\rfloor\right.$ denotes the integer part of $\left.\frac{T+1}{2}\right)$,

$$
u(k) \geq \frac{k_{0}}{T+1}\|u\|, \quad u \in\left[k_{0}, 1+T-k_{0}\right]_{\mathbf{z}}
$$

Specially, $u(k) \geq \frac{1}{T+1}\|u\|, u \in[1, T]_{\mathbf{Z}}$.

Proof (i) If $k^{*}=0$ or $T+1$, the result is clear. Now we assume that $k^{*} \in[1, T]_{\mathrm{Z}}$. Since

$$
\Delta u\left(k^{*}-1\right)=u\left(k^{*}\right)-u\left(k^{*}-1\right) \geq 0, \quad \Delta u\left(k^{*}\right)=u\left(k^{*}+1\right)-u\left(k^{*}\right) \leq 0 .
$$

We have by the monotonicity of $\Delta u(\cdot)$ that $\Delta u(k-1) \geq 0$ for $k \in\left[1, k^{*}\right]_{\mathrm{Z}}, \Delta u(k) \leq 0$ for $k \in$ $\left[k^{*}, T\right]_{\mathrm{Z}}$, which implies that $u(k) \geq 0$ holds for all $k \in[1, T]_{\mathrm{Z}}$ by the boundary conditions $u(0) \geq 0, u(T+1) \geq 0$.

(ii) For $a, b \in \mathbf{R}$ with $a<b$, let $[a, b]_{\mathbf{R}}$ and $(a, b)_{\mathbf{R}}$ denote the closed and open interval on $\mathbf{R}$, respectively. For any given $k \in[1, T+1]_{\mathbf{Z}}$, define

$$
\tilde{u}(t)=(u(k)-u(k-1))(t-k)+u(k), \quad t \in[k-1, k]_{\mathbf{R}} .
$$

Clearly, $\tilde{u}(k)=u(k)$ for $k \in[0, T+1]_{\mathbf{Z}}$ and $\tilde{u}(t)$ is continuous concave on $[0, T+1]_{\mathbf{R}}$. Let $\|\cdot\|_{C[0, T+1]_{\mathbf{R}}}$ denote the super norm in the space $C[0, T+1]_{\mathbf{R}}$. It is easy to see that $\|u\|=$ $\|u\|_{C[0, T+1]_{\mathbf{R}}}$. By the concavity of $\tilde{u}(t)$ on $[0, T+1]_{\mathbf{R}}$, we have that for any $\delta \in\left(0, \frac{T+1}{2}\right)_{\mathbf{R}}$,

$$
\tilde{u}(t) \geq \frac{\delta}{T+1}\|u\|_{C[0, T+1]_{\mathbf{R}}}, \quad t \in[\delta, T+1-\delta]_{\mathbf{R}} .
$$

which implies the expected results. 
Remark If $u(k)$ is strictly concave on $[0,1+T]_{\mathbf{Z}}$, and $u(0) \geq 0, u(T+1) \geq 0$. Then $u(k)>0$ for $k \in[1, T]_{\mathbf{Z}}$, and there exists $k^{*} \in[0,1+T]_{\mathbf{Z}}$ such that $u\left(k^{*}\right)=\|u\|, \Delta u(k-1)>0$ for $k \in\left[1, k^{*}-1\right]_{\mathrm{Z}}, \Delta u\left(k^{*}-1\right) \geq 0$, and $\Delta u(k)<0$ for $k \in\left[k^{*}, T\right]_{\mathrm{Z}}$.

Lemma 2.3 Let (B1) hold and $u$ satisfy the following difference inequality

$$
-\Delta(\phi(\Delta u(k-1))) \geq 0, \quad k \in[1, T]_{\mathrm{Z}}
$$

with $u(0) \geq 0, u(T+1) \geq 0$. Then $u(k)$ is nonnegative concave on $[0, T+1]_{\mathrm{Z}}$. Specially, if inequality (4) is strict, then $u(k)$ is strictly concave on $[0, T+1]_{\mathbf{Z}}$.

Proof Since $\Delta[\phi(\Delta u(k-1))]=\phi(\Delta u(k))-\phi(\Delta u(k-1)) \leq 0, k \in[1, T]_{\mathrm{Z}}$, we have by the monotonicity of $\phi$ that $\Delta u(k) \leq \Delta u(k-1), k \in[1, T]_{\mathrm{Z}}$, which implies that $u(k)$ is concave on $[0, T+1]_{\mathrm{Z}}$ and $u(k) \geq 0$ for $[0, T+1]_{\mathrm{Z}}$ by Lemma $2.2(\mathrm{i})$.

Lemmas 2.2 and 2.3 yield the following result.

Lemma 2.4 Let (B1) hold. Then each solution $u$ of $(1)$ is strictly concave and $u(k)>0$ for all $k \in[1, T]_{\mathrm{Z}}$

Consider the following boundary value problem

$$
\left\{\begin{array}{l}
\Delta(\phi(\Delta u(k-1)))+h(k)=0, \quad k \in[1, T]_{\mathrm{Z}} \\
u(0)=u(T+1)=0
\end{array}\right.
$$

where $h(k)>0$ for all $k \in[1, T]_{\mathrm{Z}}$ and $\phi$ satisfies (A1). It is easy to check that $u \in E$ is a solution of (5) if and only if

$$
u(k)=\sum_{s=1}^{k} \phi^{-1}\left(C_{1}+\sum_{l=s}^{T} h(l)\right), \quad k \in[0, T+1]_{\mathrm{Z}},
$$

where $C_{1}$ satisfies $u(T+1)=0$, i.e.,

$$
\sum_{k=1}^{T+1} \phi^{-1}\left(C_{1}+\sum_{l=k}^{T} h(l)\right)=0 .
$$

Since $\phi^{-1}$ is a homeomorphism from $\mathbf{R}$ onto itself, the solution $C_{1}$ of (7) is unique. Let

$$
C_{2}=-C_{1}-\lambda \sum_{l=1}^{T} h(l)
$$

Then (6) can be rewritten as follows

$$
u(k)=\sum_{s=k}^{T} \phi^{-1}\left(C_{2}+\sum_{l=1}^{s} h(l)\right), \quad k \in[0, T+1]_{\mathrm{Z}}
$$


where $C_{2}$ satisfies $u(0)=0$, i.e.,

$$
\sum_{k=0}^{T} \phi^{-1}\left(C_{2}+\sum_{l=1}^{k} h(l)\right)=0
$$

By Lemmas 2.2 and 2.3, the following result holds.

Lemma 2.5 Let (A1) hold. Assume that $u$ solves (5) and $\|u\|=u\left(k^{*}\right)$. Then

$$
C_{1}+\sum_{l \leq k^{*}}^{T} h(l) \geq 0, \quad C_{1}+\sum_{l \geq k^{*}+1}^{T} h(l) \leq 0 .
$$

Equivalently,

$$
C_{2}+\sum_{l=1}^{k \leq k^{*}-1} h(l) \leq 0, \quad C_{2}+\sum_{l=1}^{k \geq k^{*}} h(l) \geq 0
$$

Define $K=\left\{u \in E: u\right.$ is nonnegative concave on $\left.[0, T+1]_{\mathrm{z}}\right\}$. Then $K$ is a cone in $E$. Define $T_{\lambda}: K \rightarrow E$ by

$$
\left(T_{\lambda} u\right)(k)=\sum_{s=1}^{k} \phi^{-1}\left(C_{1}+\lambda \sum_{l=s}^{T} p(l) g(u(l))\right), \quad k \in[0, T+1]_{\mathbf{Z}}
$$

where $C_{1}$ is the unique solution of the following equation

$$
\sum_{k=1}^{T+1} \phi^{-1}\left(C_{1}+\lambda \sum_{l=k}^{T} p(l) g(u(l))\right)=0 .
$$

Then $T_{\lambda}(K) \subset K$ and $T_{\lambda}$ is continuous. We know that $u$ is a positive solution of (1) if and only if $T_{\lambda} u=u$ on $E$.

Lemma 2.6 Let (A1) and (A2) hold, $\Re$ be a compact subset in $(0, \infty)$. Then there exists a constant $b_{\Re}>0$ such that for all $\lambda \in \Re$ and all possible positive solutions $u$ of (1) at $\lambda$, one has $\|u\| \leq b_{\Re}$.

Proof Suppose that the conclusion is not true. Then there exists a sequence $\left\{\lambda_{n}\right\} \subset \Re$ and $u_{n}$ corresponding positive solutions of (1) at $\lambda_{n}$ such that $\left\|u_{n}\right\| \rightarrow \infty$ as $n \rightarrow \infty$. Let $\underline{\lambda}=$ $\inf \left\{\lambda_{n}\right\}, \underline{p}=\min _{k \in[1, T]_{\mathrm{Z}}} p(k)$ and $\epsilon=\frac{1}{\lambda \underline{p}}$. Since $0 \notin \Re$, we know $\underline{\lambda}>0$. By (A2), there exists $R>0$ such that $g(u)>\epsilon \phi(u)$ for all $u>R$. By the concavity of $u_{n}$ and Lemma 2.2, we know that

$$
u_{n}(k) \geq \frac{1}{T+1}\left\|u_{n}\right\|>R, \quad k \in[1, T]_{\mathrm{Z}}
$$

holds for $n$ sufficiently large. It follows that

$$
g\left(u_{n}(k)\right)>\epsilon \phi\left(u_{n}(k)\right), \quad k \in[1, T]_{\mathrm{z}} .
$$


Let $\left\|u_{n}\right\|=u_{n}\left(k^{*}\right)$. If $k^{*}>1$, then by Lemma 2.5 , we have a contradiction that

$$
\begin{aligned}
u_{n}(1) & =\phi^{-1}\left(C_{1}^{(n)}+\lambda_{n} \sum_{l=1}^{T} p(l) g\left(u_{n}(l)\right)\right) \\
& =\phi^{-1}\left(C_{1}^{(n)}+\lambda_{n}\left(\sum_{k=1}^{k^{*}-1}+\sum_{k=k^{*}}^{T}\right) p(k) g\left(u_{n}(k)\right)\right) \\
& \geq \phi^{-1}\left(\lambda_{n} \sum_{k=1}^{k^{*}-1} p(k) g\left(u_{n}(k)\right)\right) \\
& >\phi^{-1}\left(\underline{\lambda} \underline{\underline{p} \epsilon} \phi\left(u_{n}(1)\right)\right)=u_{n}(1)
\end{aligned}
$$

where $C_{1}^{(n)}$ satisfies $u_{n}(T+1)=0$. If $k^{*}=1$, then we also have a contradiction that

$$
\begin{aligned}
u_{n}(T) & =\phi^{-1}\left(C_{2}^{(n)}+\lambda_{n} \sum_{l=1}^{T} p(l) g\left(u_{n}(l)\right)\right) \\
& =\phi^{-1}\left(C_{2}^{(n)}+\lambda_{n} p(1) g\left(u_{n}(1)\right)+\lambda_{n} \sum_{k=2}^{T} p(k) g\left(u_{n}(k)\right)\right) \\
& \geq \phi^{-1}\left(\lambda_{n} \sum_{k=2}^{T} p(k) g\left(u_{n}(k)\right)\right) \\
& >\phi^{-1}\left(\underline{\lambda} \underline{\underline{\lambda}} \epsilon\left(u_{n}(T)\right)\right)=u_{n}(T)
\end{aligned}
$$

where $C_{2}^{(n)}$ satisfies $u_{n}(0)=0$. The proof is completed.

Lemma 2.7 Let (B1) hold. Then there exists $\lambda^{*}>0$ such that (1) has a positive solution at $\lambda^{*}$.

Proof Take $\alpha \equiv 0$. Then $\alpha$ is a lower solution of (1). Let $\beta$ solve the following boundary value problem

$$
\left\{\begin{array}{l}
\Delta(\phi(\Delta u(k-1)))+p(k)=0, \quad k \in[1, T]_{\mathrm{Z}} \\
u(0)=u(T+1)=0
\end{array}\right.
$$

Take $M=\max _{k \in[1, T]_{\mathbf{Z}}} f(\beta(k)), \lambda^{*}=\frac{1}{M}$. Then

$$
\Delta(\phi(\Delta \beta(k-1)))+\lambda^{*} p(k) f(\beta(k))=p(k)\left(\lambda^{*} f(\beta(k))-1\right) \leq 0, \quad k \in[1, T]_{\mathbf{z}}
$$

Therefore, $\beta$ is an upper solution of (1) at $\lambda^{*}$. By Lemma 2.1, (1) has a positive solution $u$ at $\lambda^{*}$, and the proof is done.

Lemma 2.8 Let (B1) hold. If the problem (1) has a positive solution at $\bar{\lambda}$, then (1) also has a positive solution at $\lambda$ for all $0<\lambda<\bar{\lambda}$.

Proof Let $\bar{u}$ be a positive solution of (1) at $\bar{\lambda}$ and $\lambda$ satisfy $0<\lambda<\bar{\lambda}$. Then $\bar{u}$ is an upper solution of (1) at $\lambda$ and $\alpha=0$ is a lower solution. Thus, Lemma 2.1 implies that (1) has a positive solution at $\lambda^{*}$. 
Let $\Lambda=\{\lambda \in(0, \infty)$ : the problem (1) has at least one positive solution at $\lambda\}$.

Lemma 2.9 Let (A1) and (A2) hold. Then $\Lambda$ is bounded.

Proof Suppose, on the contrary, that there exists a sequence $\left\{\lambda_{n}\right\} \subset \Lambda$ and $u_{n}$ corresponding positive solutions of (1) at $\lambda_{n}$ such that $\lambda_{n} \rightarrow \infty$ as $n \rightarrow \infty$. By (A2), there exists $\epsilon>0$ such that for all $u \geq 0, g(u) \geq \epsilon \phi(u)$. Since $\lambda_{n} \rightarrow \infty$ as $n \rightarrow \infty$, there exists $\delta>0$ satisfying $\delta p \epsilon>1$ such that $\lambda_{n}>\delta$ holds for $n$ sufficiently large. Let $\left\|u_{n}\right\|=u_{n}\left(k^{*}\right)$. Similar to the arguments in the proof of Lemma 2.6, if $k^{*}>1$, we have the following contradiction

$$
u_{n}(1)>\phi^{-1}\left(\delta \underline{p} \epsilon \phi\left(u_{n}(1)\right)\right)>u_{n}(1)
$$

and if $k^{*}=1$, we also have a contradiction that

$$
u_{n}(T)>\phi^{-1}\left(\underline{\delta} \underline{\epsilon} \phi\left(u_{n}(T)\right)\right)>u_{n}(T) .
$$

Lemma 2.10 Let (A1) and (A2) hold. Then there exists $\lambda^{*}>0$ such that $\Lambda=\left(0, \lambda^{*}\right]$.

Proof By Lemmas 2.7-2.9, $\Lambda$ is a nonempty bounded interval open at the left with the left endpoint $0 \notin \Lambda$. We only need to show that it is closed at the right. Let $\sup \Lambda=\lambda^{*}$. Then there exists a sequence $\left\{\lambda_{n}\right\} \subset \Lambda$ satisfying $\lambda_{n}<\lambda_{n+1}$ such that $\lambda_{n} \rightarrow \lambda^{*}$ as $n \rightarrow \infty$. Since $\left\{\lambda_{n}\right\}$ is bounded, Lemma 2.6 implies that there exists a constant $\aleph$ such that for all $n$ and all possible positive solutions $u_{n}$ of (1) at $\lambda_{n},\left\|u_{n}\right\| \leq \aleph$. It follows that $\left\{u_{n}\right\}$ has a convergent subsequence, say again $\left\{u_{n}\right\}$, which converges to $u^{*}$. Since $\left(\lambda_{n}, u_{n}\right)$ solves problem (1), we know that

$$
\left\{\begin{array}{l}
\Delta\left(\phi\left(\Delta u_{n}(k-1)\right)\right)+\lambda_{n} p(k) g\left(u_{n}(k)\right)=0, \quad k \in[1, T]_{\mathrm{z}}, \\
u_{n}(0)=u_{n}(T+1)=0 .
\end{array}\right.
$$

Let $n \rightarrow \infty$, we have by the continuity of $g$ as well as $\phi$ that

$$
\left\{\begin{array}{l}
\Delta\left(\phi\left(\Delta u^{*}(k-1)\right)\right)+\lambda^{*} p(k) g\left(u^{*}(k)\right)=0, \quad k \in[1, T]_{\mathrm{Z}} \\
u^{*}(0)=u^{*}(T+1)=0
\end{array}\right.
$$

which implies that $\left(\lambda^{*}, u^{*}\right)$ solves problem (1). By Lemma $2.4, u^{*}$ is positive on $[1, T]_{\mathrm{Z}}$ and $\lambda^{*} \in \Lambda$. The proof is completed.

In the succeeding arguments, we need the following well-known fixed point index theorem on cones. For proof and details, see Guo [22].

Lemma 2.11 Let $E$ be a Banach space, let $P$ be a cone in $E$, and let $\Omega$ be a bounded open set in $E$ with $0 \in \Omega$. Let $T: P \cap \bar{\Omega} \rightarrow P$ be a complete continuous operator satisfying that

$$
T x=\mu x, \quad x \in P \cap \partial \Omega \Rightarrow \mu<1 .
$$

Then $i(T, P \cap \Omega, P)=1$. 


\section{The proof of Theorem 1.1}

By Lemma 2.10, there exists $\lambda^{*}>0$ such that problem (1) has at least one positive solution for $\lambda \in\left(0, \lambda^{*}\right]$, and no solution for $\lambda>\lambda^{*}$. So we only need to show the existence of the second positive solution for $\lambda \in\left(0, \lambda^{*}\right)$. Let $u^{*}$ be the positive solution of (1) at $\lambda^{*}$, and $\lambda: 0<\lambda<\lambda^{*}$ be given. Choose a constant $M>$ such that $g\left(u^{*}(k)\right)>M$ for $k \in[1, T]_{\mathrm{Z}}$. Since $g$ is uniformly continuous on $\left[0,\left\|u^{*}\right\|+1\right]$, there exits a sufficiently small number $\epsilon>0$ such that for all $u \in E:\left\|u-u^{*}\right\| \leq \epsilon$,

$$
\left|g(u(k))-g\left(u^{*}(k)\right)\right|<\frac{\lambda^{*}-\lambda}{\lambda} M, \quad k \in[0, T+1]_{\mathbf{Z}} .
$$

Let $u_{\epsilon}^{*}(k)=u^{*}(k)+\epsilon, k \in[0, T+1]_{\mathrm{Z}}$. We claim that

$$
\Delta\left(\phi\left(\Delta u_{\epsilon}^{*}(k-1)\right)\right)+\lambda p(k) g\left(u_{\epsilon}^{*}(k)\right)<0, \quad k \in[1, T]_{\mathbf{Z}} .
$$

In fact, for $k \in[1, T]_{\mathrm{Z}}$,

$$
\begin{aligned}
\Delta & \left(\phi\left(\Delta u_{\epsilon}^{*}(k-1)\right)\right)+\lambda p(k) g\left(u_{\epsilon}^{*}(k)\right) \\
& =\Delta\left(\phi\left(\Delta u^{*}(k-1)\right)\right)+\lambda p(k) g\left(u_{\epsilon}^{*}(k)\right) \\
& =-\lambda^{*} p(k) g\left(u^{*}(k)\right)+\lambda p(k) g\left(u_{\epsilon}^{*}(k)\right) \\
& =\lambda p(k)\left[g\left(u_{\epsilon}^{*}(k)\right)-g\left(u^{*}(k)\right)\right]-\left(\lambda^{*}-\lambda\right) p(k) g\left(u^{*}(k)\right) \\
& <\left(\lambda^{*}-\lambda\right) p(k)\left(M-g\left(u^{*}(k)\right)\right)<0 .
\end{aligned}
$$

Define $\Omega=\left\{u \in E:-\epsilon<u(k)<u_{\epsilon}^{*}(k), k \in[1, T]_{\mathrm{Z}}\right\}$. Then $\Omega$ is bounded and open in $E$. Consider the following problem

$$
\left\{\begin{array}{l}
\Delta(\phi(\Delta u(k-1)))+\lambda p(k) g^{*}(u(k))=0, \quad k \in[1, T]_{\mathrm{Z}} \\
u(0)=u(T+1)=0
\end{array}\right.
$$

where

$$
g^{*}(u)= \begin{cases}g\left(u_{\epsilon}^{*}(k)\right)-\frac{u-u_{\epsilon}^{*}(k)}{1+u-u_{\epsilon}^{*}(k)}, & u>u_{\epsilon}^{*}(k), \\ g(u), & 0 \leq u \leq u_{\epsilon}^{*}(k), \\ g(0), & u<0 .\end{cases}
$$

Then $g^{*}: \mathbf{R} \rightarrow \mathbf{R}$ is continuous and bounded function. We show that all solutions $u$ of (12) satisfy $u(k)<u_{\epsilon}^{*}(k), k \in[0, T+1]_{\mathbf{Z}}$. If it is not true, then there exists $k_{0} \in[1, T]_{\mathbf{Z}}$ such that

$$
u\left(k_{0}\right)-u_{\epsilon}^{*}\left(k_{0}\right)=\max _{k \in[1, T]_{\mathbf{Z}}}\left(u(k)-u_{\epsilon}^{*}(k)\right) \geq 0 .
$$

It follows that $\Delta u\left(k_{0}-1\right) \geq \Delta u_{\epsilon}^{*}\left(k_{0}-1\right)$ and $\Delta u_{\epsilon}^{*}\left(k_{0}\right) \geq \Delta u\left(k_{0}\right)$. Thus,

$$
\Delta\left(\phi\left(\Delta u_{\epsilon}^{*}\left(k_{0}-1\right)\right)\right) \geq \Delta\left(\phi\left(\Delta u\left(k_{0}-1\right)\right)\right) .
$$

On the other hand,

$$
g^{*}\left(u\left(k_{0}\right)\right)=g\left(u_{\epsilon}^{*}\left(k_{0}\right)\right)-\frac{u\left(k_{0}\right)-u_{\epsilon}^{*}\left(k_{0}\right)}{1+u\left(k_{0}\right)-u_{\epsilon}^{*}\left(k_{0}\right)} \leq g\left(u_{\epsilon}^{*}\left(k_{0}\right)\right)
$$


and (11) implies that

$$
\Delta\left(\phi\left(\Delta u\left(k_{0}-1\right)\right)\right)=-\lambda p\left(k_{0}\right) g^{*}\left(u\left(k_{0}\right)\right) \geq-\lambda p\left(k_{0}\right) g\left(u_{\epsilon}^{*}\left(k_{0}\right)\right)>\Delta\left(\phi\left(\Delta u_{\epsilon}^{*}\left(k_{0}-1\right)\right)\right),
$$

which contradicts (13). Thus, if $u$ is a solution of (12), then $u \in \Omega$. Define $T_{\lambda}^{*}$ the same as $T_{\lambda}$ replacing $g$ by $g^{*}$. Then $T_{\lambda}^{*}: K \rightarrow K$ is continuous. Since $g^{*}$ is bounded, there exists $R_{1}>0$ such that $\left\|T_{\lambda}^{*} u\right\|<R_{1}$ for all $u \in K$. Thus, we can choose $R$ sufficiently large such that $R>R_{1}$ and $\Omega \subset B_{R}$. Here $B_{R}=\{u \in E:\|u\|<R\}$. Then by Lemma 2.11, we have $i\left(T_{\lambda}^{*}, B_{R} \cap K, K\right)=1$. Since all positive fixed points of $T_{\lambda}^{*}$ are contained in $\Omega$, and $T_{\lambda} u=u$ is equivalent to $T_{\lambda}^{*} u=u$ on $\Omega \cap K$, one has

$$
i\left(T_{\lambda}, \Omega \cap K, K\right)=i\left(T_{\lambda}^{*}, \Omega \cap K, K\right)=i\left(T_{\lambda}^{*}, B_{R} \cap K, K\right)=1 .
$$

By Lemma 2.9, there exists $\bar{\lambda}>\lambda^{*}$ such that problem (1) has no positive solution at $\bar{\lambda}$. Thus, for any open set $U$ in $E$,

$$
i\left(T_{\bar{\lambda}}, U \cap K, K\right)=0 .
$$

Take $\epsilon=\frac{1}{\lambda p}$, where $\underline{p}=\min _{k \in[1, T]_{\mathbf{Z}}} p(k)$. By (A2), there exists $L>0$ such that $g(u)>\epsilon \phi(u)$ for $u>L$. Let $\Re$ be a compact set of $(0, \infty)$ containing $\lambda$ and $\bar{\lambda}$. Then by Lemma 2.6 , we can choose $b_{\Re}>0$ sufficiently large such that all possible solutions $u$ of (1) at any $\tilde{\lambda} \in \Re$ satisfy $\|u\|<b_{\Re}$ and that $b_{\Re}>(T+1) L$ and $\Omega \subset B_{b_{\Re}}$. Here $B_{b_{\Re}}=\left\{u \in E:\|u\|<b_{\Re}\right\}$. Define $h:[0,1] \times\left(\bar{B}_{b_{\Re}} \cap K\right) \rightarrow K$ by

$$
h(t, u)=T_{\tau \bar{\lambda}+(1-\tau) \lambda}(u) .
$$

It is easy to see that $h$ is continuous on $[0,1] \times K, h(0, u)=T_{\lambda} u$ and $h(1, u)=T_{\bar{\lambda}} u$. We need to prove that $h(\tau, u) \neq u$ for all $(\tau, u) \in[0,1] \times\left(\partial \bar{B}_{b_{\Re}} \cap K\right)$. Assume that there exists $(\tau, u) \in[0,1] \times\left(\partial \bar{B}_{b_{\Re}} \cap K\right)$ such that

$$
u(k)=\sum_{s=1}^{k} \phi^{-1}\left(C_{1}^{(h)}+(\tau \bar{\lambda}+(1-\tau) \lambda) \sum_{l=s}^{T} p(l) g(u(l))\right), \quad k \in[0, T+1]_{\mathbf{Z}}
$$

where $C_{1}^{(h)}$ satisfies $u(T+1)=0$. Then by Lemma 2.2, $u(k) \geq \frac{1}{T+1}\|u\|>L$ for $k \in[1, T]_{\mathrm{Z}}$. Let $\|u\|=u\left(k^{*}\right)$. Similar to the proof of Lemma 2.6, if $k^{*}>1$, then one can get a contradiction that

$$
u(1)>\phi^{-1}((\tau \bar{\lambda}+(1-\tau) \lambda) \underline{p} \epsilon \phi(u(1)))>u(1) .
$$

If $k^{*}=1$, one can also have a contradiction. Therefore, $h(\tau, u) \neq u$ for all $(\tau, u) \in[0,1] \times$ $\left(\partial \bar{B}_{b \Re} \cap K\right)$ and $i\left(h(\tau, \cdot), B_{b_{\Re}} \cap K, K\right)$ is well defined. Thus, by the property of homotopy invariance and (15),

$$
i\left(T_{\lambda}, B_{b_{\Re}} \cap K, K\right)=i\left(T_{\bar{\lambda}}, B_{b_{\Re}} \cap K, K\right)=0 .
$$


Then by the additive property, we have from (14) and (16),

$$
i\left(T_{\lambda},\left(B_{b_{\Re}} \backslash \bar{\Omega}\right) \cap K, K\right)=-1 .
$$

Therefore, problem (1) has one positive solution in $\Omega \cap K$ and another in $\left(B_{b_{\Re}} \backslash \bar{\Omega}\right) \cap K$. The proof is completed.

\section{Competing interests}

The author declares that he has no competing interests.

\section{Author's contributions}

The author worked on the results independently.

\section{Acknowledgements}

The author is very grateful to the referees for their helpful comments. This research is supported partially by the Research Funds for the Doctoral Program of Higher Education of China (No. 20104410120001, 20114410110002), PCSIRT of China (No. IRT1226) and the Natural Science Fund of China (No. 11171078).

\section{Received: 11 June 2013 Accepted: 15 August 2013 Published: 29 August 2013}

\section{References}

1. Agarwal, RP, O'Regan, D: Boundary value problems for discrete equations. Appl. Math. Lett. 10(4), 83-89 (1997)

2. Henderson, J, Thompson, HB: Existence of multiple solutions for second-order discrete boundary value problems. Comput. Math. Appl. 43, 1239-1248 (2002)

3. Rachunkova, I, Rachunek, L: Solvability of discrete Dirichlet problem via lower and upper functions method. J. Differ. Equ. Appl., 13(5), 423-429 (2007)

4. Bai, D, Xu, Y: Nontrivial solutions of boundary value problems of second order difference equations. J. Math. Anal. Appl. 326(1), 297-302 (2007)

5. Bereanu, C, Mawhin, J, Neuve, L: Existence and multiplicity results for nonlinear second order difference equations with Dirichlet value conditions. Math. Bohem. 2, 145-160 (2006)

6. Agarwal, RP, Perera, K, O'Regan, D: Multiple positive solutions of singular discrete $p$-Laplacian problems via variational methods. Adv. Differ. Equ. 2, 93-99 (2005)

7. Cabada, A, Iannizzotto, A, Tersianc, S: Multiple solutions for discrete boundary value problems. J. Math. Anal. Appl. 356, 418-428 (2009)

8. Jiang, D, Pang, P, Agarwal, RP: Upper and lower solutions method and a superlinear singular discrete boundary value problem. Dyn. Syst. Appl. 16, 743-754 (2007)

9. Dang, H, Oppenheimer, S: Existence and uniqueness results for some nonlinear boundary value problems. J. Math. Anal. Appl. 198, 35-48 (1996)

10. Wang, H: On the number of positive solutions of nonlinear systems. J. Math. Anal. Appl. 281, 287-306 (2003)

11. Lee, E, Lee, Y: A global multiplicity result for two-point boundary value problems of $p$-Laplacian systems. Sci. China Math. 53(4), 967-984 (2010)

12. Cabada, A, Habets, P, Pouso, RL: Optimal existence conditions for $\phi$-Laplacian equations with upper and lower solutions in the reversed order. J. Differ. Equ. 166, 385-401 (2000)

13. Cabada, A, Pouso, RL: Existence results for the problem $\left(\phi\left(u^{\prime}\right)\right)^{\prime}=f\left(t, u, u^{\prime}\right)$ with nonlinear boundary conditions. Nonlinear Anal. 35, 221-231 (1999)

14. Arrázola, E, Ubilla, P: Positive solutions for the 1-dimensional generalized $p$-Laplacian involving a real parameter Proyecciones 17, 189-200 (1998)

15. Henderson, J, Wang, H: An eigenvalue problem for quasilinear systems. Rocky Mt. J. Math. 37, 215-228 (2007)

16. Lian, W, Wong, F: Existence of positive solutions for higher order generalized p-Laplacian BVPs. Appl. Math. Lett. 13, 35-43 (2000)

17. Bai, D, Chen, Y: Three positive solutions for a generalized Laplacian boundary value problem with a parameter. Appl. Math. Comput. 219, 4782-4788 (2013)

18. Cabada, A: Extremal solutions for the difference $\phi$-Laplacian problem with nonlinear functional boundary conditions Comput. Math. Appl. 42, 593-601 (2001)

19. Cabada, A, Otero-Espinar, V: Existence and comparison results for difference $\phi$-Laplacian boundary value problems with lower and upper solutions in reverse order. J. Math. Anal. Appl. 267, 501-521 (2002)

20. Bondar, K, Borkar, V, Patil, S: Existence and uniqueness results for difference $\phi$-Laplacian boundary value problems. ITB J. Sci. 43A(1), 51-58 (2011)

21. Lee, E, Lee, Y: A multiplicity result for generalized Laplacian system with multiparameters. Nonlinear Anal. 71 e366-e376 (2009)

22. Guo, D: Nonlinear Functional Analysis. Shandong Science and Technology Press, Jinan (2002) (in Chinese)

doi:10.1186/1687-1847-2013-264

Cite this article as: Bai: A global result for discrete $\phi$-Laplacian eigenvalue problems. Advances in Difference Equations 2013 2013:264 